\title{
Study of $a_{0}^{0}(980)-f_{0}(980)$ mixing from $a_{0}(1450) \rightarrow a_{0}^{0}(980) f_{0}(500) \rightarrow \pi^{+} \pi^{-} f_{0}(500)$
}

\author{
Xiao-Dong Cheng $\odot,{ }^{1, *}$ Ru-Min Wang $\odot,{ }^{2}$ and Yuan-Guo $\mathrm{Xu}^{2}$ \\ ${ }^{1}$ College of Physics and Electronic Engineering, Xinyang Normal University, \\ Xinyang 464000, People's Republic of China \\ ${ }^{2}$ College of Physics and Communication Electronics, JiangXi Normal University, \\ NanChang 330022, People's Republic of China
}

(Received 6 August 2020; accepted 24 August 2020; published 9 September 2020)

\begin{abstract}
The $a_{0}^{0}(980)-f_{0}(980)$ mixing is one of the most promising potential tools to learn about the nature of $a_{0}^{0}(980)$ and $f_{0}(980)$. Using the $f_{0}(980)-a_{0}^{0}(980)$ mixing intensity $\xi_{a f}$ measured recently at the BESIII experiment, we calculate the branching ratio of the isospin violation decay $J / \psi \rightarrow \gamma \eta_{c} \rightarrow$ $\gamma \pi^{0} a_{0}^{0}(1450) \rightarrow \gamma \pi^{0} a_{0}^{0}(980) f_{0}(500) \rightarrow \gamma \pi^{0} f_{0}(980) f_{0}(500) \rightarrow \gamma \pi^{0} \pi^{+} \pi^{-} \pi^{+} \pi^{-}$. The value of the branching ratio is found to be $O\left(10^{-6}\right)$, which can be observed with $10^{10} \mathrm{~J} / \psi$ events collected at the BESIII experiment. The narrow peak from the $f_{0}(980)-a_{0}^{0}(980)$ mixing in the $\pi^{+} \pi^{-}$mass square spectrum can also be observed. In addition, we study the nonresonant decay $a_{0}^{0}(1450) \rightarrow f_{0}(980) \pi^{+} \pi^{-}$(nonresonant), which is dominated by the $a_{0}^{0}(980)-f_{0}(980)$ mixing. We find that the nonresonant decay $a_{0}^{0}(1450) \rightarrow$ $f_{0}(980) \pi^{+} \pi^{-}$and the decay $a_{0}^{0}(1450) \rightarrow f_{0}(980) f_{0}(500)$ can be combined to measure the mixing intensity $\xi_{a f}$ in experiment. These decays are the perfect complement to the decay $\chi_{c 1} \rightarrow f_{0}(980) \pi^{0} \rightarrow$ $\pi^{+} \pi^{-} \pi^{0}$, which had been observed at the BESIII experiment, the observations of which will make the measurement of the mixing intensity $\xi_{a f}$ more precisely.
\end{abstract}

DOI: 10.1103/PhysRevD.102.054009

\section{INTRODUCTION}

The inner structure of the light scalar mesons such as $a_{0}^{0}(980)$ and $f_{0}(980)$ has been studied for over 30 years, and it is still a hot topic in particle physics. There are several proposals for the inner structure of the light scalar mesons, such as $q \bar{q}$ states, glueball, hybrid states, molecule states, tetraquark states, and the superpositions of these contents [1-11]. However, there is still no general agreement on the inner structure of $a_{0}^{0}(980)$ and $f_{0}(980)$, due to the absence of convincing evidence.

The $a_{0}^{0}(980)-f_{0}(980)$ mixing, which was first suggested theoretically in Ref. [12], is one of the most promising potential tools to learn about the nature of $a_{0}^{0}(980)$ and $f_{0}(980)$ and, therefore, has been studied extensively in various processes [13-51]. In February 2018, The BESIII Collaboration studied the $a_{0}^{0}(980)-f_{0}(980)$ mixing with the decays of $J / \psi \rightarrow \phi f_{0}(980) \rightarrow \phi a_{0}^{0}(980) \rightarrow \phi \eta \pi^{0}$ and $\chi_{c 1} \rightarrow a_{0}^{0}(980) \pi^{0} \rightarrow f_{0}(980) \pi^{0} \rightarrow \pi^{+} \pi^{-} \pi^{0}$; the signals of

\footnotetext{
*chengxd@mails.ccnu.edu.cn
}

Published by the American Physical Society under the terms of the Creative Commons Attribution 4.0 International license. Further distribution of this work must maintain attribution to the author(s) and the published article's title, journal citation, and DOI. Funded by SCOAP ${ }^{3}$. the $a_{0}^{0}(980)-f_{0}(980)$ mixing were observed with a statistical significance of larger than $5 \sigma$ for the first time. The values of the mixing intensities were measured as [52]

$$
\begin{array}{ll}
\xi_{f a}=(0.99 \pm 0.35) \times 10^{-2} & (\text { solution} 1), \\
\xi_{f a}=(0.41 \pm 0.25) \times 10^{-2} & (\text { solution} 2),
\end{array}
$$

and

$$
\xi_{a f}=(0.40 \pm 0.17) \times 10^{-2} .
$$

Here, the mixing intensities $\xi_{a f}$ and $\xi_{f a}$ are defined as

$$
\begin{aligned}
\xi_{f a} & =\frac{\mathcal{B}\left(J / \psi \rightarrow \phi f_{0}(980) \rightarrow \phi a_{0}^{0}(980) \rightarrow \phi \eta \pi^{0}\right)}{\mathcal{B}\left(J / \psi \rightarrow \phi f_{0}(980) \rightarrow \phi \pi^{+} \pi^{-}\right)}, \\
\xi_{a f} & =\frac{\mathcal{B}\left(\chi_{c 1} \rightarrow a_{0}^{0}(980) \pi^{0} \rightarrow f_{0}(980) \pi^{0} \rightarrow \pi^{+} \pi^{-} \pi^{0}\right)}{\mathcal{B}\left(\chi_{c 1} \rightarrow a_{0}^{0}(980) \pi^{0} \rightarrow \eta \pi^{0} \pi^{0}\right)} .
\end{aligned}
$$

There are two solutions for the mixing intensity $\xi_{f a}$, the recent theoretical calculation preferred to the solution-1 result [53]. The result of $\xi_{a f}$ suffers large uncertainty, and a question whether there would be a difference between the two mixing intensities $\xi_{a f}$ and $\xi_{f a}$ may be raised, so more 
precise data and more reactions are needed in both experiment and theory.

The $a_{0}(1450)$ resonance is a scalar-isovector meson and is assumed to be the conventional quark-antiquark structure based on the native quark model; the latest theoretical calculations [54-56] also confirmed this conclusion. The $B A B A R$ Collaboration performed a Dalitz plot analysis for the $\eta_{c} \rightarrow K^{+} K^{-} \pi^{0}$ and $\eta_{c} \rightarrow K^{+} K^{-} \eta$ decays and obtained the branch fraction of the $\eta_{c} \rightarrow$ $a_{0}(1450) \pi^{0} \rightarrow K^{+} K^{-} \pi^{0}$ decay relative to the $\eta_{c} \rightarrow$ $K^{+} K^{-} \pi^{0}$ mode [57]

$$
\begin{aligned}
& \frac{\mathcal{B}\left(\eta_{c} \rightarrow a_{0}(1450) \pi^{0}\right) \cdot \mathcal{B}\left(a_{0}(1450) \rightarrow K^{+} K^{-}\right)}{\mathcal{B}\left(\eta_{c} \rightarrow K^{+} K^{-} \pi^{0}\right)} \\
& =(10.2 \pm 2.5) \times 10^{-2}
\end{aligned}
$$

By combining the recent data on the branching ratio of $\eta_{c} \rightarrow K^{+} K^{-} \pi^{0}$ and $J / \psi \rightarrow \gamma \eta_{c}$ from the Particle Data Group [58]

$$
\begin{gathered}
\mathcal{B}\left(J / \psi \rightarrow \gamma \eta_{c}\right)=(1.7 \pm 0.4) \times 10^{-2}, \\
\mathcal{B}\left(\eta_{c} \rightarrow K^{+} K^{-} \pi^{0}\right)=(3.65 \pm 0.25) \times 10^{-2},
\end{gathered}
$$

and the value of the branching ratio of $a_{0}(1450) \rightarrow K^{+} K^{-}$ in Ref. [59]

$$
\mathcal{B}\left(a_{0}(1450) \rightarrow K^{+} K^{-}\right)=(4.61 \pm 0.61) \times 10^{-2},
$$

we find that the branching ratio of $J / \psi \rightarrow \gamma \eta_{c} \rightarrow$ $\gamma a_{0}(1450) \pi^{0}$ can reach the order of $10^{-3}$. Based on the data samples of $10^{10} \mathrm{~J} / \psi$ events collected in the BESIII experiment [60-63], about $10^{7} a_{0}(1450)$ mesons can be produced through decays $J / \psi \rightarrow \gamma \eta_{c} \rightarrow \gamma a_{0}(1450) \pi^{0}$; this large $a_{0}(1450)$ sample at the BESIII experiment will make it possible to investigate the properties of $a_{0}(1450)$ meson and study the related physics.

In this paper, we investigate the isospin breaking decay $\eta_{c} \rightarrow a_{0}(1450) \pi^{0} \rightarrow a_{0}^{0}(980) f_{0}(500) \pi^{0} \rightarrow \pi^{+} \pi^{-} f_{0}(500) \pi^{0}$ produced via $J / \psi \rightarrow \gamma \eta_{c}$. We predict the branching ratio of this reaction by using the recent measurements at the BESIII experiment and calculate the distribution of the $\pi^{+} \pi^{-}$mass square spectrum near the $K \bar{K}$ thresholds. We also discuss the $a_{0}(1450) \rightarrow f_{0}(980) \pi^{+} \pi^{-}$(nonresonant) $\rightarrow$ $\pi^{+} \pi^{-} \pi^{+} \pi^{-}$decay process, which is realized mainly via the $a_{0}^{0}(980)-f_{0}(980)$ mixing.

\section{DATA ON THE DECAY}

The $\quad \eta_{c} \rightarrow \pi^{0} a_{0}(1450) \rightarrow \pi^{0} a_{0}^{0}(980) f_{0}(500) \rightarrow$ $\pi^{0} \pi^{+} \pi^{-} f_{0}(500)$ decay violates the isospin symmetry; it can proceed via the $a_{0}^{0}(980)-f_{0}(980)$ mixing. In this process, the mixing intensity $\xi_{a f}$ is given as

$$
\xi_{a f}=\frac{\mathcal{B}\left(\eta_{c} \rightarrow \pi^{0} a_{0}(1450) \rightarrow \pi^{0} a_{0}^{0}(980) f_{0}(500) \rightarrow \pi^{0} f_{0}(980) f_{0}(500) \rightarrow \pi^{0} \pi^{+} \pi^{-} \pi^{+} \pi^{-}\right)}{\mathcal{B}\left(\eta_{c} \rightarrow \pi^{0} a_{0}(1450) \rightarrow \pi^{0} a_{0}^{0}(980) f_{0}(500) \rightarrow \pi^{0} \eta \pi^{0} f_{0}(500) \rightarrow \pi^{0} \eta \pi^{0} \pi^{+} \pi^{-}\right)}
$$

Here, $\mathcal{B}\left(\eta_{c} \rightarrow \pi^{0} a_{0}(1450) \rightarrow \pi^{0} a_{0}^{0}(980) f_{0}(500) \rightarrow \pi^{0} \eta \pi^{0} f_{0}(500) \rightarrow \pi^{0} \eta \pi^{0} \pi^{+} \pi^{-}\right)$is the branching ratio of the $\eta_{c} \rightarrow$ $\pi^{0} a_{0}(1450) \rightarrow \pi^{0} a_{0}^{0}(980) f_{0}(500) \rightarrow \pi^{0} \eta \pi^{0} f_{0}(500) \rightarrow \pi^{0} \eta \pi^{0} \pi^{+} \pi^{-}$decay

$$
\begin{aligned}
& \mathcal{B}\left(\eta_{c} \rightarrow \pi^{0} a_{0}(1450) \rightarrow \pi^{0} a_{0}^{0}(980) f_{0}(500) \rightarrow \pi^{0} \eta \pi^{0} f_{0}(500) \rightarrow \pi^{0} \eta \pi^{0} \pi^{+} \pi^{-}\right) \\
& \quad=\mathcal{B}\left(\eta_{c} \rightarrow \pi^{0} a_{0}(1450)\right) \cdot \mathcal{B}\left(a_{0}(1450) \rightarrow a_{0}^{0}(980) f_{0}(500)\right) \cdot \mathcal{B}\left(a_{0}^{0}(980) \rightarrow \eta \pi^{0}\right) \cdot \mathcal{B}\left(f_{0}(500) \rightarrow \pi^{+} \pi^{-}\right) .
\end{aligned}
$$

By combining Eqs. (5) and (7), one can obtain

$$
\begin{aligned}
& \mathcal{B}\left(\eta_{c} \rightarrow \pi^{0} a_{0}(1450)\right) \cdot \mathcal{B}\left(a_{0}(1450) \rightarrow K^{+} K^{-}\right) \\
& \quad=(3.72 \pm 0.96) \times 10^{-3} .
\end{aligned}
$$

The ratio of the branching ratio of $a_{0}(1450) \rightarrow$ $a_{0}^{0}(980) f_{0}(500)$ to $a_{0}(1450) \rightarrow K^{+} K^{-}$has been presented in Ref. [59],

$$
\frac{\mathcal{B}\left(a_{0}(1450) \rightarrow a_{0}^{0}(980) f_{0}(500)\right)}{\mathcal{B}\left(a_{0}(1450) \rightarrow K^{+} K^{-}\right)}=8.24 \pm 2.72
$$

so we can predict the branching ratio of the decay chain $\eta_{c} \rightarrow a_{0}(1450) \pi^{0} \rightarrow a_{0}^{0}(980) f_{0}(500) \pi^{0}$ as

$$
\begin{aligned}
& \mathcal{B}\left(\eta_{c} \rightarrow \pi^{0} a_{0}(1450)\right) \cdot \mathcal{B}\left(a_{0}(1450) \rightarrow a_{0}^{0}(980) f_{0}(500)\right) \\
& \quad=(3.06 \pm 1.28) \times 10^{-2} .
\end{aligned}
$$

From Refs. $[1,64,65]$, we can obtain the branching ratios $\mathcal{B}\left(f_{0}(500) \rightarrow \pi^{+} \pi^{-}\right), \quad \mathcal{B}\left(f_{0}(980) \rightarrow \pi^{+} \pi^{-}\right), \quad$ and $\mathcal{B}\left(a_{0}^{0}(980) \rightarrow \eta \pi^{0}\right)$,

$$
\begin{gathered}
\mathcal{B}\left(f_{0}(500) \rightarrow \pi^{+} \pi^{-}\right)=0.67, \\
\mathcal{B}\left(f_{0}(980) \rightarrow \pi^{+} \pi^{-}\right)=0.50_{-0.09}^{+0.07}, \\
\mathcal{B}\left(a_{0}^{0}(980) \rightarrow \eta \pi^{0}\right)=0.845 \pm 0.017 .
\end{gathered}
$$

Substituting Eqs. (14), (16), and (13) into Eq. (10), one could predict the branching ratio of the decay chain 
$\eta_{c} \rightarrow \pi^{0} a_{0}(1450) \rightarrow \pi^{0} a_{0}^{0}(980) f_{0}(500) \rightarrow \pi^{0} \eta \pi^{0} f_{0}(500) \rightarrow$ $\pi^{0} \eta \pi^{0} \pi^{+} \pi^{-}$as

$$
\begin{aligned}
& \mathcal{B}\left(\eta_{c} \rightarrow \pi^{0} a_{0}(1450) \rightarrow \pi^{0} a_{0}^{0}(980) f_{0}(500)\right. \\
&\left.\quad \rightarrow \pi^{0} \eta \pi^{0} f_{0}(500) \rightarrow \pi^{0} \eta \pi^{0} \pi^{+} \pi^{-}\right) \\
&=(1.74 \pm 0.72) \times 10^{-2}
\end{aligned}
$$

Combining this equation with Eq. (2) and using Eq. (9), we then obtain the branching ratio $\mathcal{B}\left(\eta_{c} \rightarrow \pi^{0} a_{0}(1450) \rightarrow\right.$ $\left.\pi^{0} a_{0}^{0}(980) f_{0}(500) \rightarrow \pi^{0} f_{0}(980) f_{0}(500) \rightarrow \pi^{0} \pi^{+} \pi^{-} \pi^{+} \pi^{-}\right)$ as $(0.70 \pm 0.41) \times 10^{-4}$. Adding this value and Eq. (6) together, we can readily obtain

$$
\begin{aligned}
& \mathcal{B}\left(J / \psi \rightarrow \gamma \eta_{c} \rightarrow \gamma \pi^{0} a_{0}(1450) \rightarrow \gamma \pi^{0} a_{0}^{0}(980) f_{0}(500)\right. \\
& \left.\quad \rightarrow \gamma \pi^{0} f_{0}(980) f_{0}(500) \rightarrow \gamma \pi^{0} \pi^{+} \pi^{-} \pi^{+} \pi^{-}\right) \\
& =(1.19 \pm 0.75) \times 10^{-6} .
\end{aligned}
$$

Obviously, we believe this decay will be marginally detected in the $e^{+} e^{-}$colliders in view of the large database of the BESIII Collaboration.

\section{MECHANISM RESPONSIBLE FOR THE DECAY}

As for the decay $a_{0}(1450) \rightarrow a_{0}^{0}(980) f_{0}(500) \rightarrow$ $f_{0}(980) f_{0}(500) \rightarrow \pi^{+} \pi^{-} f_{0}(500)$, the amplitude is proportional to the mixing of the $a_{0}^{0}(980)$ and $f_{0}(980)$ resonances, which is caused by the mass difference of the $K^{+} K^{-}$and $K^{0} \bar{K}^{0}$ intermediate state. The diagram of $f_{0}(980)$ production in the $a_{0}(1450) \rightarrow a_{0}^{0}(980) f_{0}(500) \rightarrow$ $f_{0}(980) f_{0}(500) \rightarrow \pi^{+} \pi^{-} f_{0}(500)$ reaction is shown in Fig 1, so the decay amplitude from this process can be written as

$$
\begin{aligned}
& \mathcal{M}\left(a_{0}(1450) \rightarrow a_{0}^{0}(980) f_{0}(500)\right. \\
& \left.\quad \rightarrow f_{0}(980) f_{0}(500) \rightarrow \pi^{+} \pi^{-} f_{0}(500)\right) \\
& =\mathcal{M}_{a_{0}(1450) a_{0}^{0}(980) f_{0}(500)} \cdot \frac{\Pi_{a_{0}^{0} f_{0}}\left(q^{2}\right)}{D_{a_{0}^{0}}\left(q^{2}\right) D_{f_{0}}\left(q^{2}\right)-\Pi_{a_{0}^{0} f_{0}}^{2}\left(q^{2}\right)} \\
& \quad \cdot g_{f_{0}(980) \pi^{+} \pi^{-}},
\end{aligned}
$$

where $q^{2}=\left(p_{\pi^{+}}+p_{\pi^{-}}\right)^{2}$, and $a_{0}^{0}$ and $f_{0}$ are, respectively, the shorthand of $a_{0}^{0}(980)$ and $f_{0}(980) . \mathcal{M}_{a_{0}(1450) a_{0}^{0}(980) f_{0}(500)}$ is the invariant amplitude for the decay $a_{0}(1450) \rightarrow$ $a_{0}^{0}(980) f_{0}(500)$,

$$
\begin{aligned}
\mathcal{B}\left(a_{0}(1450) \rightarrow\right. & \left.a_{0}^{0}(980) f_{0}(500)\right) \\
= & \left|\mathcal{M}_{a_{0}(1450) a_{0}^{0}(980) f_{0}(500)}\right|^{2} \\
& \cdot \frac{f\left(m_{a_{0}(1450)}, m_{a_{0}^{0}(980)}, m_{f_{0}(500)}\right)}{16 \pi \Gamma_{a_{0}(1450)} m_{a_{0}(1450)}^{3}} .
\end{aligned}
$$

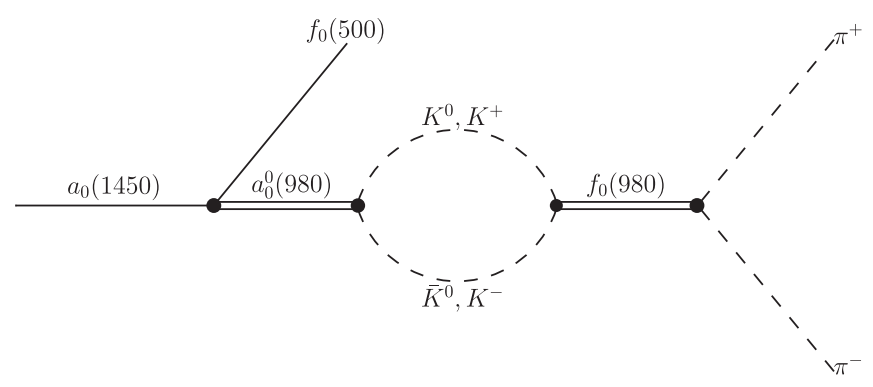

FIG. 1. Feynman diagram for the reaction $a_{0}(1450) \rightarrow$ $a_{0}^{0}(980) f_{0}(500) \rightarrow f_{0}(980) f_{0}(500) \rightarrow \pi^{+} \pi^{-} f_{0}(500)$.

Hereinafter, $m_{r}$ denotes the mass of resonance $r[r=$ $\left.f_{0}(980), a_{0}^{0}(980), f_{0}(500), a_{0}(1450), \eta, \pi^{0}, \pi^{+}, K^{0}, K^{+}\right]$and $\Gamma_{r}$ with $r=a_{0}^{0}(980), f_{0}(500), f_{0}(980), a_{0}(1450)$ denotes the width of the resonance. The function $f(x, y, z)$ is defined as

$f(x, y, z)=\sqrt{x^{4}+y^{4}+z^{4}-2 x^{2} y^{2}-2 x^{2} z^{2}-2 y^{2} z^{2}}$.

$g_{f_{0}(980) \pi^{+} \pi^{-}}$is the coupling constant of $f_{0}(980)$ with $\pi^{+} \pi^{-}$ and can be extracted from the branching ratio of the $f_{0}(980) \rightarrow \pi^{+} \pi^{-}$decay

$$
\begin{aligned}
\mathcal{B}\left(f_{0}(980) \rightarrow \pi^{+} \pi^{-}\right)= & \left|g_{f_{0}(980) \pi^{+} \pi^{-}}\right|^{2} \\
& \cdot \frac{f\left(m_{f_{0}(980)}, m_{\pi^{+}}, m_{\pi^{+}}\right)}{16 \pi \Gamma_{f_{0}(980)} m_{f_{0}(980)}^{3}} .
\end{aligned}
$$

The $a_{0}^{0}(980)-f_{0}(980)$ mixing amplitude $\Pi_{a_{0}^{0} f_{0}}\left(q^{2}\right)$ has the following form $[44,66]$ :

$$
\begin{aligned}
\Pi_{a_{0}^{0} f_{0}}\left(q^{2}\right)= & \frac{g_{a_{0}^{0}(980) K^{+} K^{-}} g_{f_{0}(980) K^{+} K^{-}}}{16 \pi} \\
& \times\left[i\left(R_{K^{+} K^{-}}\left(q^{2}\right)-R_{K^{0} \bar{K}^{0}}\left(q^{2}\right)\right)\right. \\
& -\frac{R_{K^{+} K^{-}}\left(q^{2}\right)}{\pi} \ln \frac{1+R_{K^{+} K^{-}}\left(q^{2}\right)}{1-R_{K^{+} K^{-}}\left(q^{2}\right)} \\
& \left.+\frac{R_{K^{0} \bar{K}^{0}}\left(q^{2}\right)}{\pi} \ln \frac{1+R_{K^{0} \bar{K}^{0}}\left(q^{2}\right)}{1-R_{K^{0} \bar{K}^{0}}\left(q^{2}\right)}\right],
\end{aligned}
$$

where $g_{a_{0}^{0}(980) K^{+} K^{-}}$and $g_{f_{0}(980) K^{+} K^{-}}$is the coupling constant of $K^{+} K^{-}$with $a_{0}^{0}(980)$ and $f_{0}(980)$, respectively. For $q^{2} \geq$ $4 m_{a}^{2}\left[a=K^{+}, K^{0}\right], R_{a a}\left(q^{2}\right)=\sqrt{1-4 m_{a}^{2} / q^{2}}$, if $q^{2} \leq 4 m_{a}^{2}$, then $R_{a a}\left(q^{2}\right)$ should be replaced by $i \sqrt{4 m_{a}^{2} / q^{2}-1}$. In Eq. (19), $D_{r}\left(q^{2}\right)$ is the inverse propagator of the unmixed resonance $r$,

$$
D_{r}\left(q^{2}\right)=q^{2}-m_{r}^{2}-\sum_{a b}\left[\operatorname{Re} \Pi_{r}^{a b}\left(m_{r}^{2}\right)-\Pi_{r}^{a b}\left(q^{2}\right)\right] .
$$


For $r=a_{0}^{0}(980), a b=\left(\eta \pi^{0}, K^{+} K^{-}, K^{0} \bar{K}^{0}\right)$; for $r=f_{0}(980)$, $a b=\left(\pi^{+} \pi^{-}, \pi^{0} \pi^{0}, K^{+} K^{-}, K^{0} \bar{K}^{0}\right) . \Pi_{r}^{a b}$ denote the diagonal matrix of the polarization operator of the resonance $r$ corresponding to the one loop contribution from the two-particle intermediate $a b$ states, it is a piecewise function, and its expressions in the different $q^{2}$ regions are displayed in Eqs. (18-20) of Ref. [67]. Making use of Eqs. (19), (20), and (22), it is then straightforward to obtain

$$
\begin{aligned}
& \frac{d \Gamma\left(a_{0}(1450) \rightarrow a_{0}^{0}(980) f_{0}(500) \rightarrow f_{0}(980) f_{0}(500) \rightarrow \pi^{+} \pi^{-} f_{0}(500)\right)}{d q^{2}}=\mathcal{B}\left(f_{0}(980) \rightarrow \pi^{+} \pi^{-}\right) \\
& \cdot \mathcal{B}\left(a_{0}(1450) \rightarrow a_{0}^{0}(980) f_{0}(500)\right) \cdot\left|\frac{\Pi_{a_{0}^{0} f_{0}}\left(q^{2}\right)}{D_{a_{0}^{0}}\left(q^{2}\right) D_{f_{0}}\left(q^{2}\right)-\Pi_{a_{0}^{0} f_{0}}^{2}\left(q^{2}\right)}\right|^{2} \cdot \varphi_{S},
\end{aligned}
$$

where $\varphi_{S}$ is the relevant phase-space factor

$$
\varphi_{S}=\frac{\Gamma_{a_{0}(1450)} \Gamma_{f_{0}(980)} m_{f_{0}(980)}^{3}}{\pi q^{2}} \cdot \frac{\bar{f}\left(m_{a_{0}(1450)}, m_{f_{0}(500)}, \sqrt{q^{2}}\right)}{\bar{f}\left(m_{a_{0}(1450)}, m_{f_{0}(500)}, m_{a_{0}^{0}(980)}\right)} \cdot \frac{f\left(\sqrt{q^{2}}, m_{\pi^{+}}, m_{\pi^{+}}\right)}{f\left(m_{f_{0}(980)}, m_{\pi^{+}}, m_{\pi^{+}}\right)} .
$$

Since the decay width of the $f_{0}(500)$ resonance is large, we use the functions $\bar{f}\left(m_{a_{0}(1450)}, m_{f_{0}(500)}, z\right)\left[z=\sqrt{q^{2}}, m_{a_{0}^{0}(980)}\right]$ in Eq. (26), which can be obtained by the functions $f\left(m_{a_{0}(1450)}, m_{f_{0}(500)}, z\right)$ weighed with the $f_{0}(500)$ resonant distribution, i.e.,

$$
\bar{f}\left(m_{a_{0}(1450)}, m_{f_{0}(500)}, z\right)=\int_{4 m_{\pi^{+}}^{2}}^{\left(a_{0}(1450)-z\right)^{2}} \rho(m) f\left(m_{a_{0}(1450)}, m, z\right) d m^{2},
$$

where $\rho(m)$ is the spectral density and can be approximated as

$$
\rho(m)=\frac{1}{\pi} \frac{m \Gamma_{f_{0}(500)}}{\left(m^{2}-m_{f_{0}(500)}^{2}\right)^{2}+\left(m \Gamma_{f_{0}(500)}\right)^{2}} .
$$

\begin{tabular}{|c|c|}
\hline$m_{\pi^{+}}=139.6 \mathrm{MeV}[58]$ & $m_{\pi^{0}}=135 \mathrm{MeV}[58]$ \\
\hline$m_{K^{+}}=493.7 \mathrm{MeV}[58]$ & $m_{K^{0}}=497.6 \mathrm{MeV}[58]$ \\
\hline$m_{\eta}=547.9 \mathrm{MeV}[58]$ & $m_{\eta^{\prime}}=(957.8 \pm 0.1) \mathrm{MeV}[58]$ \\
\hline$m_{f_{0}(980)}=(0.99 \pm 0.02) \mathrm{GeV}[58]$ & $\Gamma_{f_{0}(980)}=0.074 \mathrm{GeV}[68]$ \\
\hline$m_{a_{0}^{0}(980)}=(0.98 \pm 0.02) \mathrm{GeV}[58]$ & $\Gamma_{a_{0}^{0}(980)}=(0.092 \pm 0.008) \mathrm{GeV}[58]$ \\
\hline$m_{a_{0}(1450)}=(1.474 \pm 0.019) \mathrm{GeV}[58]$ & $\Gamma_{a_{0}(1450)}=(0.265 \pm 0.013) \mathrm{GeV}[58]$ \\
\hline$m_{f_{0}(500)}=(0.475 \pm 0.075) \mathrm{GeV}[58]$ & $\Gamma_{f_{0}(500)}=(0.55 \pm 0.15) \mathrm{GeV}[58]$ \\
\hline \multicolumn{2}{|c|}{$g_{a_{0}^{0}(980) \eta \pi^{0}}=2.43 \mathrm{GeV}[1,64]$} \\
\hline$g_{a_{0}^{0}(980) K^{+} K^{-}}=(2.76 \pm 0.46) \mathrm{GeV}[69,70]$ & $g_{a_{0}^{0}(980) K^{0} \bar{K}^{0}}=(2.76 \pm 0.46) \mathrm{GeV}[69,70]$ \\
\hline$g_{f_{0}(980) \pi^{+} \pi^{-}}=1.39 \mathrm{GeV}[1,64]$ & $g_{f_{0}(980) \pi^{0} \pi^{0}}=0.98 \mathrm{GeV}[1,64]$ \\
\hline$g_{f_{0}(980) K^{+} K^{-}}=3.17 \mathrm{GeV}[44]$ & $g_{f_{0}(980) K^{0} \bar{K}^{0}}=3.17 \mathrm{GeV}$ \\
\hline
\end{tabular}

By multiplying both sides of Eq. (25) by $\mathcal{B}\left(J / \psi \rightarrow \gamma \eta_{c}\right)$ and $\mathcal{B}\left(\eta_{c} \rightarrow a_{0}(1450) \pi^{0}\right)$, one can obtain

$$
\begin{aligned}
& \mathcal{B}\left(J / \psi \rightarrow \gamma \eta_{c} \rightarrow \gamma a_{0}(1450) \pi^{0}\right) \cdot \frac{d \Gamma\left(a_{0}(1450) \rightarrow a_{0}^{0}(980) f_{0}(500) \rightarrow \pi^{+} \pi^{-} f_{0}(500)\right)}{d q^{2}} \\
& =\mathcal{B}\left(J / \psi \rightarrow \gamma \eta_{c}\right) \cdot \mathcal{B}\left(\eta_{c} \rightarrow a_{0}(1450) \pi^{0}\right) \cdot \mathcal{B}\left(a_{0}(1450) \rightarrow a_{0}^{0}(980) f_{0}(500)\right) \\
& \quad \cdot \mathcal{B}\left(f_{0}(980) \rightarrow \pi^{+} \pi^{-}\right) \cdot\left|\frac{\Pi_{a_{0}^{0} f_{0}}\left(q^{2}\right)}{D_{a_{0}^{0}}\left(q^{2}\right) D_{f_{0}}\left(q^{2}\right)-\Pi_{a_{0}^{0} f_{0}}^{2}\left(q^{2}\right)}\right|^{2} \cdot \varphi_{S},
\end{aligned}
$$

TABLE I. Properties of the resonances. 


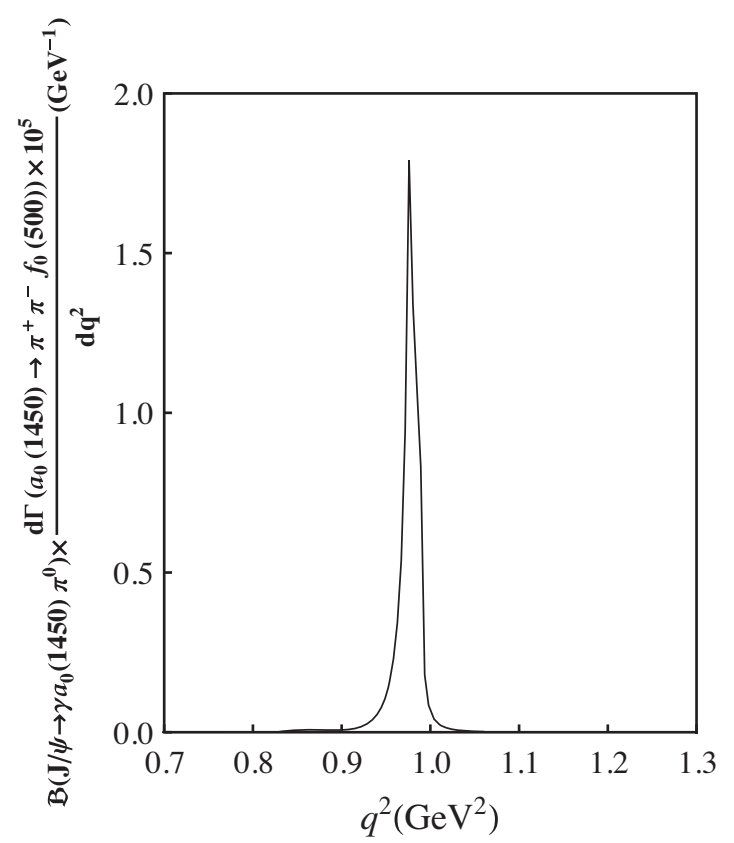

FIG. 2. The distribution of the $\pi^{+} \pi^{-}$mass square spectrum $\left[q^{2}=\left(p_{\pi^{+}}+p_{\pi^{-}}\right)^{2}\right]$ for the decay $J / \psi \rightarrow \gamma \eta_{c} \rightarrow \gamma \pi^{0} a_{0}^{0}(1450) \rightarrow$ $\gamma \pi^{0} a_{0}^{0}(980) f_{0}(500) \rightarrow \gamma \pi^{0} \pi^{+} \pi^{-} f_{0}(500)$.

With the value of the parameters that are listed in Table I and substituting Eqs. (6), (13), and (15) into Eq. (29), we can obtain the distribution curve of the $\pi^{+} \pi^{-}$mass square spectrum for the decay $J / \psi \rightarrow \gamma \eta_{c} \rightarrow \gamma \pi^{0} a_{0}^{0}(1450) \rightarrow$ $\gamma \pi^{0} a_{0}^{0}(980) f_{0}(500) \rightarrow \gamma \pi^{0} \pi^{+} \pi^{-} f_{0}(500)$, which is presented in Fig. 2. Here, we note that the narrow peak from the $a_{0}^{0}(980)-f_{0}(980)$ mixing can be clearly observed in this figure. The physical range of $q^{2}$ for $a_{0}^{0}(1450) \rightarrow$ $\pi^{+} \pi^{-} f_{0}(500)$ is $4 m_{\pi^{+}}^{2} \leq q^{2} \leq\left(m_{a_{0}(1450)}-m_{f_{0}(500)}\right)^{2}$. By integrating over the variable $q^{2}$, we finally obtain the following value of the branching ratio:

$$
\begin{gathered}
\mathcal{B}\left(J / \psi \rightarrow \gamma \eta_{c} \rightarrow \gamma \pi^{0} a_{0}^{0}(1450) \rightarrow \gamma \pi^{0} a_{0}^{0}(980) f_{0}(500)\right. \\
\left.\quad \rightarrow \gamma \pi^{0} \pi^{+} \pi^{-} f_{0}(500)\right)=\left(1.31_{-0.67}^{+0.65}\right) \times 10^{-6} .
\end{gathered}
$$

Here, we combine the uncertainties of the branching ratios involved in the calculation, the decay width of $a_{0}(1450)$, and the mass and the decay width of $f_{0}(500)$ to determine the final error of the above branching ratio. Adding Eqs. (14) and (30) together, we then arrive at

$$
\begin{aligned}
& \mathcal{B}\left(J / \psi \rightarrow \gamma \eta_{c} \rightarrow \gamma \pi^{0} a_{0}^{0}(1450) \rightarrow \gamma \pi^{0} a_{0}^{0}(980) f_{0}(500)\right. \\
& \left.\quad \rightarrow \gamma \pi^{0} \pi^{+} \pi^{-} \pi^{+} \pi^{-}\right)=\left(0.88_{-0.45}^{+0.44}\right) \times 10^{-6} .
\end{aligned}
$$

\section{NONRESONANT DECAY $\boldsymbol{a}_{\mathbf{0}}^{\mathbf{0}}(\mathbf{1 4 5 0}) \rightarrow$ $f_{0}(980) \pi^{+} \pi^{-}$}

In experiment, the resonant $f_{0}(500)$ is reconstructed by the $f_{0}(500) \rightarrow \pi^{+} \pi^{-}$decay. If we apply the selection criteria that restrict the invariant mass of $\pi^{+} \pi^{-}$to the $f_{0}(500)$ mass window, the background channel $a_{0}^{0}(1450) \rightarrow$ $f_{0}(980) \pi^{+} \pi^{-}$(nonresonant) cannot be removed because of the large width of the resonant $f_{0}(500)$. Fortunately, however, the nonresonant decay $a_{0}^{0}(1450) \rightarrow f_{0}(980) \pi^{+} \pi^{-}$ violates isospin invariant or $C$ symmetry; the violation of the isospin invariant is caused by the $a_{0}^{0}(980)-f_{0}(980)$ mixing.

In the isospin limit, the wave function of the two pions system can be written as $[71,72]$

$$
\begin{gathered}
(\pi \pi)_{I=0}^{I_{3}=0}=\frac{\sqrt{3}}{3}\left|\pi^{+}\right\rangle\left|\pi^{-}\right\rangle+\frac{\sqrt{3}}{3}\left|\pi^{-}\right\rangle\left|\pi^{+}\right\rangle-\frac{\sqrt{3}}{3}\left|\pi^{0}\right\rangle\left|\pi^{0}\right\rangle \\
(\pi \pi)_{I=1}^{I_{3}=0}=\frac{1}{\sqrt{2}}\left|\pi^{+}\right\rangle\left|\pi^{-}\right\rangle-\frac{1}{\sqrt{2}}\left|\pi^{-}\right\rangle\left|\pi^{+}\right\rangle \\
(\pi \pi)_{I=2}^{I_{3}=0}=\frac{\sqrt{6}}{6}\left|\pi^{+}\right\rangle\left|\pi^{-}\right\rangle+\frac{\sqrt{6}}{6}\left|\pi^{-}\right\rangle\left|\pi^{+}\right\rangle+\frac{\sqrt{6}}{3}\left|\pi^{0}\right\rangle\left|\pi^{0}\right\rangle
\end{gathered}
$$

In the above equations, we can see that the $C$ parity of $(\pi \pi)_{I=0}^{I_{3}=0},(\pi \pi)_{I=1}^{I_{3}=0}$, and $(\pi \pi)_{I=2}^{I_{3}=0}$ are $+1,-1$, and +1 , respectively. As for the nonresonant decay $a_{0}^{0}(1450) \rightarrow$ $f_{0}(980) \pi^{+} \pi^{-}$, if isospin is conserved, the two pions system has $I=1, I_{3}=0$, so the $C$ parity of the two pions system is -1 . As a consequence, $C\left(f_{0}(980)(\pi \pi)_{I=1}^{I_{3}=0}\right)=$ $-\left(f_{0}(980)(\pi \pi)_{I=1}^{I_{3}=0}\right)$, while it is $C=+1$ for $a_{0}^{0}(1450)$; therefore this decay violates $C$. If the nonresonant decay $a_{0}^{0}(1450) \rightarrow f_{0}(980) \pi^{+} \pi^{-}$violates isospin, then the two pions system has $I=2, I_{3}=0$ or $I=0, I_{3}=0$. In these cases, the $C$ parity of the two pions system is +1 . As a result, the $C$ parity of the system of $f_{0}(980)$ and the two pions is +1 . Meanwhile, the $C$ parity of $a_{0}^{0}(1450)$ is also +1 , so we can easily achieve that this decay conserves $C$ if it violates isospin. In a word, the nonresonant decay $a_{0}^{0}(1450) \rightarrow f_{0}(980) \pi^{+} \pi^{-}$violates $C$ or $I$. Because $C$ violation is only known to occur in weak interaction, the contribution from $C$ violation is much smaller than that from the isospin violation, which can occur in electromagnetic interaction, so the contribution from $C$ violation can be neglected. The nonresonant decay $a_{0}^{0}(1450) \rightarrow$ $f_{0}(980) \pi^{+} \pi^{-}$is determined mainly by the contribution of the isospin symmetry breaking process, which is caused by the $a_{0}^{0}(980)-f_{0}(980)$ mixing, so the nonresonant decay $a_{0}^{0}(1450) \rightarrow f_{0}(980) \pi^{+} \pi^{-}$and the decay $a_{0}^{0}(1450) \rightarrow$ $f_{0}(980) f_{0}(500)$ can be combined to measure the mixing intensity $\xi_{a f}$ in experiment.

\section{PROSPECTS FOR THE MEASUREMENT AT THE BESIII EXPERIMENT}

As for the decay $J / \psi \rightarrow \gamma \eta_{c} \rightarrow \gamma \pi^{0} a_{0}^{0}(1450) \rightarrow$ $\gamma \pi^{0} a_{0}^{0}(980) f_{0}(500) \rightarrow \gamma \pi^{0} f_{0}(980) f_{0}(500) \rightarrow \gamma \pi^{0} \pi^{+} \pi^{-} \times$ $\pi^{+} \pi^{-}$, there are four intermediate states, i.e., $\eta_{c}, a_{0}^{0}(1450)$, 
$f_{0}(500)$, and $f_{0}(980)$. Because of the narrow peak near the $K \bar{K}$ thresholds in the $\pi^{+} \pi^{-}$invariant mass spectrum, the event selection criteria for the $f_{0}(980)$ candidates has high efficiency. As discussed in Sec. IV, the selection criteria that constrain the invariant mass of $\pi^{+} \pi^{-}$to the $f_{0}(500)$ mass window also has high efficiency when both the nonresonant decay $a_{0}^{0}(1450) \rightarrow f_{0}(980) \pi^{+} \pi^{-}$and the decay $a_{0}^{0}(1450) \rightarrow$ $f_{0}(980) f_{0}(500)$ are combined. In addition, the $\pi^{0}$ final state is reconstructed through the decay $\pi^{0} \rightarrow \gamma \gamma$, for which the branching ratio is $(98.82 \pm 0.03) \%$ [58], so the final states of the decay $J / \psi \rightarrow \gamma \eta_{c} \rightarrow \gamma \pi^{0} a_{0}^{0}(1450) \rightarrow$ $\gamma \pi^{0} a_{0}^{0}(980) f_{0}(500) \rightarrow \gamma \pi^{0} f_{0}(980) f_{0}(500) \rightarrow \gamma \pi^{0} \pi^{+} \pi^{-} \pi^{+} \pi^{-}$ contain three photons and four charged tracks. After considering all of the above, we assume that the efficiency for $J / \psi \rightarrow \gamma \eta_{c} \rightarrow \gamma \pi^{0} a_{0}^{0}(1450) \rightarrow \gamma \pi^{0} a_{0}^{0}(980) f_{0}(500) \rightarrow$ $\gamma \pi^{0} f_{0}(980) f_{0}(500) \rightarrow \gamma \pi^{0} \pi^{+} \pi^{-} \pi^{+} \pi^{-}$is $3 \%$ after the final selection [61,73-75], so the branching ratio $\times$ efficiency factor of this decay can reach about $3.0 \times 10^{-8}$. The BESIII experiment will produce $10 \times 10^{9} \mathrm{~J} / \psi$ events [61-63,76], so about 300 events should be observed in the corresponding signal region. Therefore, the isospin breaking decay $J / \psi \rightarrow \gamma \eta_{c} \rightarrow \gamma \pi^{0} a_{0}^{0}(1450) \rightarrow \gamma \pi^{0} a_{0}^{0}(980) f_{0}(500) \rightarrow$ $\gamma \pi^{0} f_{0}(980) f_{0}(500) \rightarrow \gamma \pi^{0} \pi^{+} \pi^{-} \pi^{+} \pi^{-}$may be used to study the $a_{0}^{0}(980)-f_{0}(980)$ mixing and determine the value of $\xi_{\text {af }}$ exactly.

\section{CONCLUSIONS}

In summary, the large $a_{0}(1450)$ sample, which can be produced through the decays $J / \psi \rightarrow \gamma \eta_{c} \rightarrow \gamma a_{0}(1450) \pi^{0}$ and reconstructed with the $a_{0}(1450) \rightarrow \omega \pi \pi$ or $a_{0}(1450) \rightarrow$ $a_{0}(980) \pi \pi$ decays at the BESIII experiment, will allow study of the properties of $a_{0}(1450)$ resonance in more detail and investigation of the related physics. Using the $f_{0}(980)-a_{0}^{0}(980)$ mixing intensity $\xi_{a f}$ measured recently at the BESIII experiment [52], we investigate the $f_{0}(980)-$ $a_{0}^{0}(980)$ mixing through the isospin violation decay $J / \psi \rightarrow$ $\gamma \eta_{c} \rightarrow \gamma \pi^{0} a_{0}^{0}(1450) \rightarrow \gamma \pi^{0} a_{0}^{0}(980) f_{0}(500) \rightarrow \gamma \pi^{0} f_{0}(980) \times$ $f_{0}(500) \rightarrow \gamma \pi^{0} \pi^{+} \pi^{-} \pi^{+} \pi^{-}$. We find that the branching ratio for the decay can reach up to the order of $10^{-6}$, which might be hopefully measurable with $10^{10} \mathrm{~J} / \psi$ events collected at the BESIII experiment. We also observe the narrow peak from the $f_{0}(980)-a_{0}^{0}(980)$ mixing in the $\pi^{+} \pi^{-}$ mass spectrum. The related decay $a_{0}^{0}(1450) \rightarrow$ $f_{0}(980) \pi^{+} \pi^{-}$(nonresonant), which is dominated by the contribution of the isospin symmetry breaking process, can be combined with the decay $a_{0}^{0}(1450) \rightarrow$ $a_{0}^{0}(980) f_{0}(500) \rightarrow f_{0}(980) f_{0}(500) \rightarrow \pi^{+} \pi^{-} \pi^{+} \pi^{-} \quad$ to study the $f_{0}(980)-a_{0}^{0}(980)$ mixing in experiment. These decays could be complementary to the decay $\chi_{c 1} \rightarrow f_{0}(980) \pi^{0} \rightarrow \pi^{+} \pi^{-} \pi^{0}$, which has been observed at the BESIII experiment [52], the observations of which will make the measurement of the mixing intensity $\xi_{a f}$ more precisely, thus enhancing the understanding of the nature of the light scalar mesons.

\section{ACKNOWLEDGMENTS}

The work was supported by the National Natural Science Foundation of China (Contract No. 11675137).
[1] H. Y. Cheng, C. K. Chua, and K. C. Yang, Phys. Rev. D 73, 014017 (2006).

[2] J. D. Weinstein and N. Isgur, Phys. Rev. D 27, 588 (1983).

[3] J. D. Weinstein and N. Isgur, Phys. Rev. Lett. 48, 659 (1982).

[4] J. D. Weinstein and N. Isgur, Phys. Rev. D 41, 2236 (1990).

[5] R. L. Jaffe, Phys. Rev. D 15, 267 (1977); 15, 281 (1977).

[6] K. S. Kim and H. Kim, Eur. Phys. J. C 77, 435 (2017).

[7] J. Berlin, A. Abdel-Rehim, C. Alexandrou, M. Dalla Brida, M. Gravina, and M. Wagner, Proc. Sci., LATTICE2014 (2014) 104 [arXiv:1410.8757].

[8] C. Amsler and F. E. Close, Phys. Lett. B 353, 385 (1995).

[9] C. Amsler and F. E. Close, Phys. Rev. D 53, 295 (1996).

[10] C. Amsler, Phys. Lett. B 541, 22 (2002).

[11] S. G. Gorishnii, A. L. Kataev, and S. A. Larin, Phys. Lett. 135B, 457 (1984); A. L. Kataev, Yad. Fiz. 68, 597 (2005) [Phys. At. Nucl. 68, 567 (2005)].

[12] N. N. Achasov, S. A. Devyanin, and G. N. Shestakov, Phys. Lett. 88B, 367 (1979).
[13] N. N. Achasov, S. A. Devyanin, and G. N. Shestakov, Yad. Fiz. 33, 1337 (1981) [Sov. J. Nucl. Phys. 33, 715 (1981)].

[14] N. N. Achasov and G. N. Shestakov, Phys. Rev. D 56, 212 (1997).

[15] O. Krehl, R. Rapp, and J. Speth, Phys. Lett. B 390, 23 (1997).

[16] B. Kerbikov and F. Tabakin, Phys. Rev. C 62, 064601 (2000).

[17] F. E. Close and A. Kirk, Phys. Lett. B 489, 24 (2000).

[18] A. E. Kudryavtsev and V. E. Tarasov, Pis'ma Zh. Eksp. Teor. Fiz. 72, 589 (2000) [JETP Lett. 72, 410 (2000)].

[19] V. Y. Grishina, L. A. Kondratyuk, M. Buescher, W. Cassing, and H. Stroher, Phys. Lett. B 521, 217 (2001).

[20] F. E. Close and A. Kirk, Phys. Lett. B 515, 13 (2001).

[21] A. E. Kudryavtsev, V. E. Tarasov, J. Haidenbauer, C. Hanhart, and J. Speth, Phys. Rev. C 66, 015207 (2002).

[22] L. A. Kondratyuk, E. L. Bratkovskaya, V. Y. Grishina, M. Buescher, W. Cassing, and H. Stroher, Yad. Fiz. 66, 155 (2003) [Phys. At. Nucl. 66, 152 (2003)]. 
[23] N. N. Achasov and A. V. Kiselev, Phys. Lett. B 534, 83 (2002).

[24] N. N. Achasov and G. N. Shestakov, Phys. Rev. Lett. 92, 182001 (2004).

[25] V. Y. Grishina, L. A. Kondratyuk, M. Buescher, and W. Cassing, Eur. Phys. J. A 21, 507 (2004).

[26] N. N. Achasov and G. N. Shestakov, Phys. Rev. D 70, 074015 (2004).

[27] J. J. Wu, Q. Zhao, and B. S. Zou, Phys. Rev. D 75, 114012 (2007).

[28] J. J. Wu and B. S. Zou, Phys. Rev. D 78, 074017 (2008).

[29] C. Hanhart, B. Kubis, and J. R. Pelaez, Phys. Rev. D 76, 074028 (2007).

[30] M. Ablikim et al. (BESIII Collaboration), Phys. Rev. D 83, 032003 (2011).

[31] J. J. Wu, X. H. Liu, Q. Zhao, and B. S. Zou, Phys. Rev. Lett. 108, 081803 (2012).

[32] F. Aceti, W. H. Liang, E. Oset, J. J. Wu, and B. S. Zou, Phys. Rev. D 86, 114007 (2012).

[33] L. Roca, Phys. Rev. D 88, 014045 (2013).

[34] V. E. Tarasov, W. J. Briscoe, W. Gradl, A. E. Kudryavtsev, and I. I. Strakovsky, Phys. Rev. C 88, 035207 (2013).

[35] T. Sekihara and S. Kumano, Phys. Rev. D 92, 034010 (2015).

[36] F. Aceti, J. M. Dias, and E. Oset, Eur. Phys. J. A 51, 48 (2015).

[37] N. N. Achasov, A. A. Kozhevnikov, and G. N. Shestakov, Phys. Rev. D 92, 036003 (2015).

[38] W. Wang, Phys. Lett. B 759, 501 (2016).

[39] N. N. Achasov, A. A. Kozhevnikov, and G. N. Shestakov, Phys. Rev. D 93, 114027 (2016).

[40] N. N. Achasov and G. N. Shestakov, Phys. Rev. D 96, 036013 (2017).

[41] N. N. Achasov and G. N. Shestakov, Phys. Rev. D 96, 016027 (2017).

[42] S. Sakai, E. Oset, and W. H. Liang, Phys. Rev. D 96, 074025 (2017).

[43] M. Bayar and V. R. Debastiani, Phys. Lett. B 775, 94 (2017).

[44] N. N. Achasov and G. N. Shestakov, Phys. Rev. D 96, 091501 (2017).

[45] W. H. Liang, S. Sakai, J. J. Xie, and E. Oset, Chin. Phys. C 42, 044101 (2018).

[46] N. Achasov and G. Shestakov, Usp. Fiz. Nauk 189, 3 (2019).

[47] N. Achasov and G. Shestakov, EPJ Web Conf. 212, 03002 (2019).

[48] W. H. Liang, H. X. Chen, E. Oset, and E. Wang, Eur. Phys. J. C 79, 411 (2019).

[49] R. Molina, J. J. Xie, W. H. Liang, L. S. Geng, and E. Oset, Phys. Lett. B 803, 135279 (2020).

[50] N. Achasov, arXiv:2002.01354.

[51] N. Achasov, A. Kiselev, and G. Shestakov, Phys. Rev. D 102, 016022 (2020).
[52] M. Ablikim et al. (BESIII Collaboration), Phys. Rev. Lett. 121, 022001 (2018).

[53] T. M. Aliev and S. Bilmis, Eur. Phys. J. A 54, 147 (2018).

[54] N. Mathur, A. Alexandru, Y. Chen, S. J. Dong, T. Draper, I. Horvath, F. X. Lee, K. F. Liu, S. Tamhankar, and J. B. Zhang, Phys. Rev. D 76, 114505 (2007).

[55] W. J. Lee and D. Weingarten, Phys. Rev. D 61, 014015 (1999); M. Gockeler, R. Horsley, H. Perlt, P. E. Rakow, G. Schierholz, A. Schiller, and P. Stephenson, Phys. Rev. D 57, 5562 (1998); S. Kim and S. Ohta, Nucl. Phys. B, Proc. Suppl. 53, 199 (1997); A. Hart, C. McNeile, and C. Michael (UKQCD Collaboration), Nucl. Phys. B, Proc. Suppl. 119, 266 (2003); T. Burch, C. Gattringer, L. Y. Glozman, C. Hagen, C. Lang, and A. Schafer, Phys. Rev. D 73, 094505 (2006).

[56] H. Y. Han, X. G. Wu, H. B. Fu, Q. L. Zhang, and T. Zhong, Eur. Phys. J. A 49, 78 (2013).

[57] J. Lees et al. (BABAR Collaboration), Phys. Rev. D 89, 112004 (2014).

[58] M. Tanabashi et al. (Particle Data Group), Phys. Rev. D 98, 030001 (2018).

[59] D. Bugg, Phys. Rev. D 78, 074023 (2008).

[60] M. Ablikim et al. (BESIII Collaboration), Chin. Phys. C 44, 040001 (2020).

[61] D. M. Asner et al., Int. J. Mod. Phys. A 24, 499 (2009).

[62] H. B. Li, Front. Phys. (Beijing) 12, 121301 (2017).

[63] I. I. Bigi, X. W. Kang, and H. B. Li, Chin. Phys. C 42, 013101 (2018).

[64] H. Y. Cheng, C. K. Chua, K. C. Yang, and Z. Q. Zhang, Phys. Rev. D 87, 114001 (2013).

[65] M. Ablikim et al. (BESIII Collaboration), Phys. Rev. Lett. 122, 062001 (2019).

[66] N. N. Achasov and A. V. Kiselev, Phys. Rev. D 98, 096009 (2018).

[67] X. D. Cheng, H. B. Li, R. M. Wang, and M. Z. Yang, Phys. Rev. D 99, 014024 (2019).

[68] M. Ablikim et al. (BESIII Collaboration), Phys. Rev. D 99, 012014 (2019).

[69] M. Ablikim et al. (BESIII Collaboration), Phys. Rev. D 95, 032002 (2017).

[70] M. Ablikim et al. (BESIII Collaboration), Phys. Rev. Lett. 121, 081802 (2018).

[71] M. I. Buchoff, J. W. Chen, and A. Walker-Loud, Phys. Rev. D 79, 074503 (2009).

[72] H. B. Li, J. Phys. G 36, 085009 (2009).

[73] M. Ablikim et al. (BESIII Collaboration), Phys. Rev. D 86, 092009 (2012).

[74] M. Ablikim et al. (BESIII Collaboration), Phys. Rev. D 91, 052017 (2015).

[75] M. Ablikim et al. (BESIII Collaboration), Phys. Rev. Lett. 118, 012001 (2017).

[76] S. s. Fang, A. Kupsc, and D. h. Wei, Chin. Phys. C 42, 042002 (2018). 